\title{
Atendimento oftalmológico dos recém-nascidos examinados nas maternidades públicas em Manaus
}

\author{
Ophthalmological evaluations of the newborn \\ at the public maternities in Manaus
}

José Cavalcanti Campos Júnior ${ }^{1}$

\section{Resumo}

Objetivo: Determinar o tipo e a frequência de diagnóstico ocular dos recém-nascidos atendidos no ambulatório, UCI e UTI neonatais das Maternidades Públicas Estaduais na cidade de Manaus (AM). Métodos:Tratou-se de um estudo retrospectivo dos prontuários dos recém-nascidos, obtidos nas Maternidades Públicas Estaduais no município de Manaus, estado do Amazonas. Foram incluídos todos os pacientes avaliados durante trinta meses (setembro de 2005 a março de 2008). O trabalho foi submetido e aprovado pelas direções das unidades de saúde, que permitiram a execução do estudo sem restrições. Resultados: Do total dos 4.591 atendimentos realizados, 1880 (40,9\%) foram provenientes da Maternidade Balbina Mestrinho, enquanto que 1.360 (29,6\%) foram atendidos na Maternidade Ana Braga, 788 (17,2\%) na Maternidade Nazira Daou, 315 (6,9\%) na Maternidade Azilda Marreiro e 248 (5,4\%) na Maternidade da Alvorada. Em relação ao tipo de diagnóstico, encontramos RN Normais em $2.462(53,6 \%)$ casos e $2.129(46,4 \%)$ casos de RN com alterações oculares - 69,8\% (ROP), 18,9\% (Conjuntivites), 4,1\% (Catarata), 3,6\% (Estrabismo), 2,5\% (Uveítes), 0,8\% (anormalidades congênitas) e $0,3 \%$ (Outros). Conclusão: As alterações oculares foram encontradas em quase metade dos pacientes: ROP, conjuntivites, catarata, estrabismo, uveítes.

Descritores: Oftalmopatias/diagnóstico; Saúde ocular; Recém-nascido

\footnotetext{
${ }^{1}$ Pós-graduando (Doutorado) em Doenças Tropicais e Infecciosas pela Fundação de Medicina Tropical do Amazonas e Universidade do Estado do Amazonas - UEA - Manaus (AM), Brasil; Oftalmologista das Maternidades Públicas Estaduais - Manaus (AM), Brasil;

Trabalho realizado nas Maternidades Públicas do Estado do Amazonas Ana Braga, Balbina Mestrinho, Azilda Marreiro e Alvorada Manaus (AM), Brasil.
}

Recebido para publicação em: 2/12/2009 - Aceito para publicação em 13/4/2010 


\section{INTRODUÇÃO}

$\mathbf{S}$ egundo dados da Organização Mundial de Saúde (OMS) existem no mundo mais de 20 milhões de cegos, sendo que $2 / 3$ destes casos são determinados por causas preveníveis ${ }^{(1)}$. Em 1992, estimativas mundiais apontavam para a alta cifra de 1,5 milhão o número de cegos menores de 16 anos, e destes, em torno de $90 \%$ viviam em países em desenvolvimento. ${ }^{(2,3)}$

Os transtornos no desenvolvimento da visão afetam o processo de aprendizagem da criança e sua integridade, tanto física como psíquica, sendo fundamental a prevenção e sua identificação precoce. ${ }^{(4)}$ Os programas de saúde pública em oftalmologia devem priorizar as ações relacionadas à prevenção da cegueira e da incapacidade visual, promoção de saúde ocular, organização de assistência oftalmológica e reabilitação de deficientes visuais. ${ }^{(3,5)}$

A avaliação oftalmológica criteriosa dos recémnascidos tem sido uma constante nas maternidades brasileiras. O teste do olhinho, ou do reflexo vermelho, possibilita uma triagem de doenças oculares que afetam a transparência dos meios (catarata, retinoblastoma, Doença de Coats, etc.) e que consequentemente, podem provocar deficiências visuais e até cegueira.

Apesar de ter sido descrita há mais de 50 anos, a Retinopatia da Prematuridade (ROP) se tornou uma das maiores causas de cegueira infantil (definida pela OMS como cegueira, ocorrendo até os 15 anos de idade) nos países desenvolvidos, em função da maior sobrevivência de recém-nascidos prematuros (RNP) com menor peso de nascimento e com menor idade gestacional. ${ }^{(6)}$

O exame oftalmológico inicial deve ser realizado sob oftalmoscopia binocular indireta e dilatação das pupilas, entre a $4^{\mathrm{a}}$ e a $6^{\mathrm{a}}$ semana de vida, em todos os recém-nascidos com peso igual ou menor do que 1500 gramas e/ou com idade gestacional igual ou inferior a 32 semanas. $\mathrm{O}$ acompanhamento das crianças com ou sem retinopatia identificada deverá seguir periodicamente até a normalização da vascularização da retina temporal na Zona III, o que poderá tardar até mais do que os primeiros seis meses de vida e deverá ser mantido pelos dois primeiros anos para a prevenção da ambliopia e do estrabismo e para a correção das altas ametropias relacionadas com a prematuridade. ${ }^{(6)}$

Define-se catarata infantil a opacificação do cristalino que reduz a visão em indivíduos de 0 a 15 anos. ${ }^{(7)}$ Apresenta incidência de 1/2000 nascidos vivos e 10 novos casos/milhão população/ano. Estima-se que ela seja responsável por $10-38,8 \%$ de toda a cegueira prevenível e tratável na infância (AV corrigida inferior a 20/400 ou 0,05 no melhor olho em indivíduos menores de 16 anos, segundo a Organização Mundial de Saúde) em crianças de todo o mundo. ${ }^{(8)}$ A prevalência em crianças é de $1 \mathrm{a}$ 4/ 10.000 nos países em desenvolvimento e aproximadamente 0,1 a 0,4/10.000 nos países desenvolvidos. ${ }^{(9)}$ Considera-se que 40 a $50 \%$ dos portadores de catarata infantil apresentam baixa visão. ${ }^{(7)}$

A toxoplasmose é a maior causa de uveítes do polo posterior do olho. ${ }^{(10)} \mathrm{A}$ retinocoroidite é a manifestação mais importante da doença e a lesão pode se apresentar ao exame fundoscópico de uma forma ativa ou cicatrizada, e está associada à considerável perda de campo visual quando ocorre próximo ao nervo óptico. ${ }^{(11,12)}$

A doença é principalmente adquirida através da ingestão de cistos presentes em carnes cruas ou mal cozidas, especialmente suínas, e através de frutas e verduras mal lavadas contendo os cistos ${ }^{(9,10)}$. Outra via de transmissão é a transplacentária, na qual se a gestante adquirir a primo infecção durante a gestação, o feto será infectado, sendo que $85 \%$ destes desenvolvem retinocoroidite durante a infância ou adolescência. ${ }^{(11,12)}$ O diagnóstico de toxoplasmose ocular é frequentemente feito somente pelo aspecto clínico da lesão.

A conjuntivite neonatal é uma condição potencialmente séria, tanto pelos efeitos locais ao olho, quanto pelo risco de disseminação sistêmica. Os fatores predisponentes às conjuntivites neonatais são decorrentes de parto vaginal, asfixia neonatorum e ruptura prolongada da membrana amniótica. ${ }^{(13)}$

A esotropia congênita, geralmente deve-se a hipóxia ou anóxia que ocorre durante o parto, com lesão do sistema nervoso central, podendo se associar ao quadro de paralisia cerebral e ambliopia. ${ }^{(14)}$

O Estado do Amazonas, com dimensão continental e 62 municípios, possui em Manaus, apenas cinco maternidades públicas:Ana Braga,Balbina Mestrinho,Nazira Daou, Azilda Marreiro e Alvorada. Até o ano de 2005, não dispunham de nenhum oftalmologista para o diagnóstico e tratamento de patologias oculares dos recém-nascidos (RNs). Após a elaboração de projeto específico, o autor iniciou a prestação dos serviços junto a estas instituições.

O estudo foi realizado com o objetivo de identificar a frequência e o tipo de diagnóstico ocular encontrado nos pacientes recém-nascidos examinados no ambulatório,UCI (Unidade de Cuidados Intermediários) e UTI (Unidade de Tratamento Intensivo) neonatais das Maternidades Públicas Estaduais na cidade de Manaus, orientando o seguimento e tratamentos necessários o mais precoce possível.

\section{MÉTOdos}

Tratou-se de um estudo retrospectivo realizado através dos prontuários médicos dos recém-nascidos existentes nas Maternidades Públicas Estaduais, no mu- 


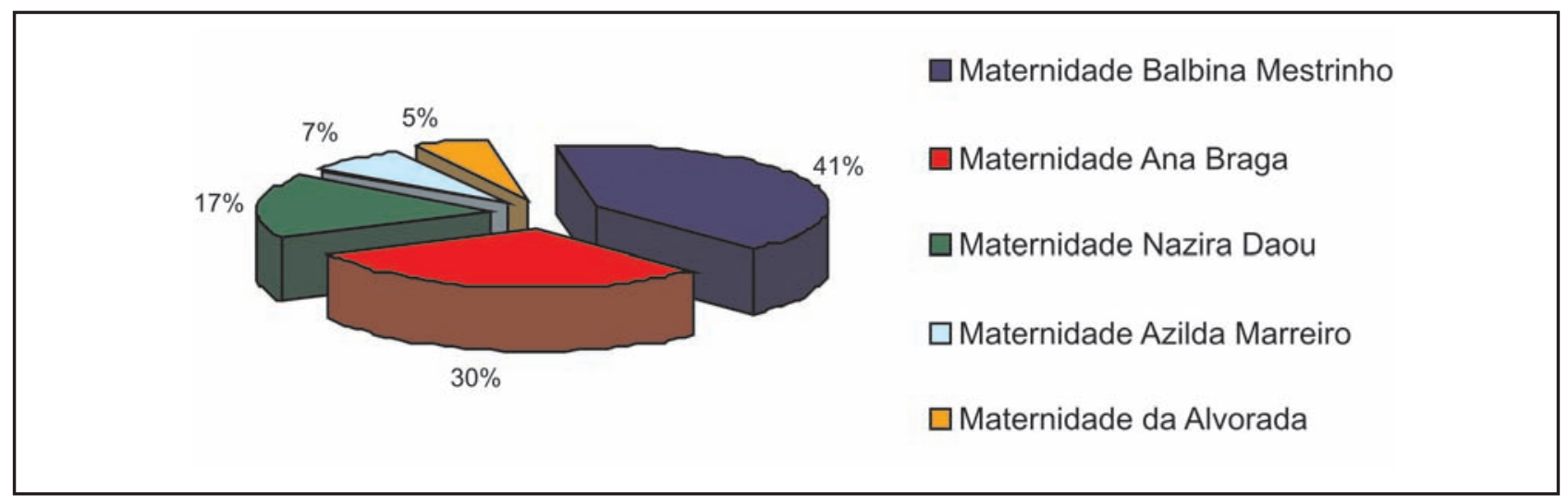

Figura 1: Distribuição dos pacientes atendidos nas maternidades estaduais

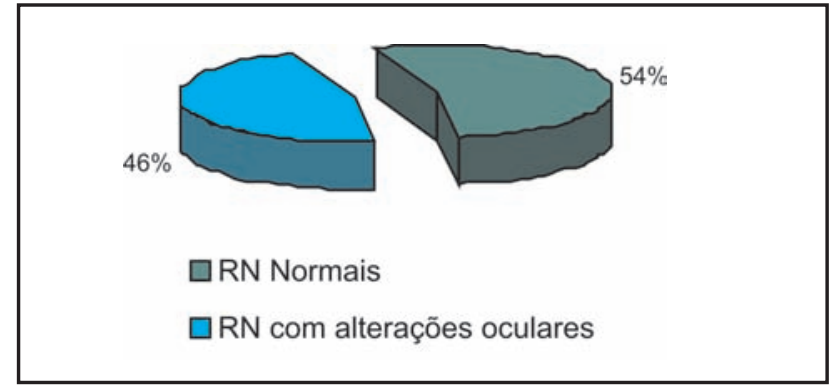

Figura 2: Distribuição dos RNs quanto ao acometimento ocular

nicípio de Manaus, Estado do Amazonas.

Os dados obtidos foram consolidados em planilha eletrônica e representados em tabelas e gráficos. Para estatística descritiva será utilizada a frequência relativa.

Foram incluídos no estudo todos os pacientes avaliados durante trinta meses (setembro de 2005 a março de 2008). O trabalho foi submetido e aprovado pelas direções das unidades de saúde, que permitiram sem restrições a execução do estudo.

\section{Resultados}

Do total dos 4.591 atendimentos realizados ( $\mathrm{Fi}-$ gura 1), 1880 (40,9\%) foram provenientes da maternidade Balbina Mestrinho, enquanto que 1360 (29,6\%) foram atendidos na maternidade Ana Braga, 788 $(17,2 \%)$ na maternidade Nazira Daou, 315 (6,9\%) na maternidade Azilda Marreiro e 248 (5,4\%) na maternidade da Alvorada.

Em relação ao acometimento ocular e o tipo de diagnóstico (Figuras 2 e 3), encontramos RNs normais em $2462(53,6 \%)$ casos, e $2129(46,4 \%)$ casos de RN com alterações oculares, sendo 69,8 \% de ROP, $18,9 \%$ com conjuntivites, 4,1\% (Catarata), 3,6\% (Estrabismo), 2,5\% (Uveítes), $0,8 \%$ (anormalidades congênitas) e 0,3 $\%$ (Outros).

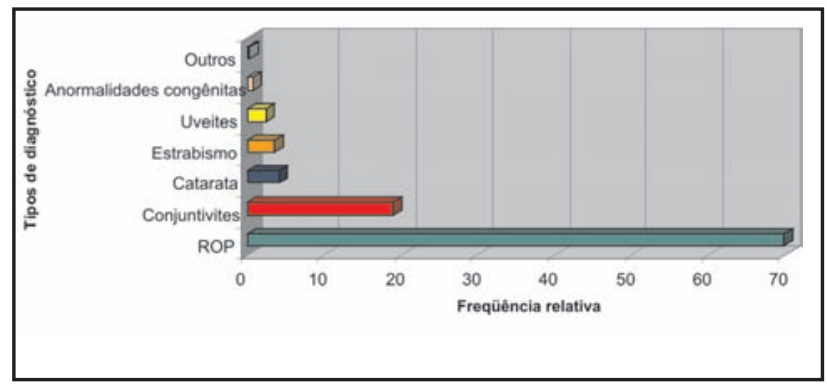

Figura 3: Distribuição dos RNs atendidos nas maternidades segundo o tipo de diagnóstico.

\section{DiscusSÃo}

Sabemos que no recém-nascido, o sistema nervoso central e, consequentemente o sistema visual, é pouco desenvolvido. Todos nós nascemos com baixa visão ${ }^{(15)}$. A partir do nascimento há evolução orgânica e funcional do sistema visual se utilizado adequadamente. Qualquer fator que impeça o desenvolvimento normal da visão pode causar alterações anatômicas e funcionais do sistema visual, levando à baixa visão. ${ }^{(16,17)}$

Nos países em desenvolvimento, os escassos recursos destinados à área de saúde devem atender a prioridades múltiplas e distintas, nem sempre privilegiando programas preventivos. Ressalta a escassez de informações na literatura nacional quanto ao exame oftalmológico do recém-nato, visando à identificação precoce de doenças oculares. ${ }^{18)}$

A catarata congênita constitui a causa mais frequente de cegueira evitável na infância, podendo atingir um porcentual de 10 a $15 \%$. Neste trabalho, encontramos em pouco mais de 4\% dos RNs. Assim, o diagnóstico precoce da catarata congênita, bem como a realização da facectomia e correção da ametropia em tempo hábil determinam a resposta visual do indivíduo.

A proporção de cegueira causada por ROP é muito influenciada pelo nível de cuidado neonatal 
(disponibilidade de recursos humanos, equipamentos, acesso e qualidade de atendimento), assim como pela existência de programas eficazes de triagem e tratamento. Por conseguinte, existe uma grande variabilidade de ocorrência da doença em países desenvolvidos e em desenvolvimento. ${ }^{(6)}$ Neste trabalho, observamos dentre os RNs, com alteração ocular $(46,4 \%)$, cerca de $70 \%$ dos casos, algum estágio de ROP, ressaltando que todas as maternidades são unidades de referência de tratamento intensivo para bebês de alto risco.

Em relação às uveites, encontrou-se em 2,5\% dos RNs, sendo a lesão retinocoroideana da toxoplasmose a manifestação mais importante da doença, em especial ao afetar a mácula, pois leva a perda permanente da capacidade visual, implicando em detrimento da qualidade de vida, com restrições ocupacionais, econômicas, sociais e psicológicas.

Neste contexto, a assistência oftalmológica aos RNs nas maternidades do SUS no Amazonas iniciou-se com um trabalho, objetivando o maior grau de resolutividade possível, onde a maioria dos atendimentos necessitou de tratamento clínico; os de intervenção cirúrgica (cataratas, estrabismos e ROP) foram encaminhados para unidades de saúde capacitada de recursos cirúrgicos para a resolução dos casos.

\section{Abstract}

Purpose: Determine the type and frequency of diagnosis of newborn seen in the ambulatories, ICU and ITU at the Public Maternities in Manaus. Methods: We present a retrospective study done at the Public Maternities in Manaus, Amazon state. We include all patients seen during thirty months (september 2005 to march 2008). The study was submitted and approved by the head of health units that allowed the execution of the study without restrictions. Results: Of the total 4.591 patients seen, 1.880 (40,9\%) were from at the Balbina Mestrinho Maternity, while 1.360 (29,6\%) were seen at the Ana Braga Maternity, 788 (17,2\%) at the Nazira Daou Maternity, 315 (6,9\%) Azilda Marreiro Maternity and 248 (5,4\%) at the Alvorada Maternity. The type of diagnoses were found: Normal Newborn in $2.462(53,6 \%)$ cases e $2.129(46,4 \%)$ cases of Ocular alterations newborn - $69,8 \%$ cases of ROP, 18,9\% cases of conjunctivitis, 4,1\% cataract, 3,6\% cases of strabismus, 2,5\% uveitis, $0,8 \%$ ocular congenital abnormalities. Conclusions:Almost half of patients showed the ocular alterations: ROP, conjunctivitis, cataract, strabismus, and uveitis.

Infant, newborn

\section{RefERÊNCIAS}

1. Adam Netto A, Peres SO. Catarata na infância - estudo de 106 casos. Rev Bras Oftalmol. 1998;57(12):903-8.

2. Foster A, Gilbert C. Epidemiology of childhood blindness. Eye (Lond). 1992;6(Pt 2):173-6.

3. Brito PR, Veitzman S. Causas de cegueira e baixa visão e crianças. Arq Bras Oftalmol. 2000;63(1):49-54.

4. Lucena AR, Cantanhede TM, Trigueiro SA, Tavares S, Ventura LO. Frequência e causas da ambliopia em pacientes assistidos na Fundação Altino Ventura- Recife-PE. Rev Bras Oftalmol. 2001;60(1):50-4.

5. Temporini ER. Pesquisa de oftalmologia em Saúde Pública: considerações metodológica sobre fatores humanos. Arq Bras Oftalmol. 1991;54(6):279-81.

6. Gilbert C. Retinopathy of prematurity: epidemiology. J Comm Eye Health. 1997;10(22):22-4.

7. Curso de Salud Ocular Comunitaria Pro Vision 2. In: Manual de Salud Ocular Comunitaria. London: Escuela de Salud Publica y Medicina Tropical; 2002. p.32-6.

8. Arieta C, José NK. Catarata congênita: dificuldades no tratamento. Arq Bras Oftalmol. 1987;50(3):116-9.

9. Murta J. Catarata pediatrica. Coimbra: Ediliber; 1998.

10. Smith RE, Nozik RA. Uveitis. A clinical approach to diagnosis and management. 2nd ed. Baltimore: Williams \& Wilkins; 1989.

11. Oréfice F, Boratto LM, organizadores. Caderno de uveítes. São Paulo: Alcon Laboratórios do Brasil; 1994. Uveítes posteriores.

12. Stanford MR, Tomlin EA, Comyn O, Holland K, Pavesio C. The visual field in toxoplasmic retinochoroiditis. Br J Ophthalmol. 2005; 89(7): 812-4.

13. Iroha EO, Kesah CN, Egri-Okwaji MTC, Odugbemi TO. Bacterial eye infection in neonates, a prospective study in a neonatal unit. West Afr J Med. 1998;17(3):168-72.

14. Almeida HC, Curi R. Manual de estrabismo. Rio de Janeiro: [s.n.]; 1997.

15. Hyvarinen L. Vision in children normal and anormal. Ontario: The Canadian Deaf-Blind \& Rubella Association;1988. 64p.

16. Schimiti RB, Costa VP, Gregui MJ, Kara-José N, Temporini ER. Prevalence of refractive errors and ocular disorders in preschool and schoolchildren of Ibiporã - PR, Brazil (1989 to 1996). Arq Bras Oftalmol. 2001; 64(5): 379-84.

17. Garcia CA, Andrade LL, Nobre GF, Gomes AH, Oréfice F. Prevalência de alterações fundoscópicas em estudantes na cidade de Natal/Brasil. Rev Bras Oftalmol. 2004; 63(11-12): 523-7.

18. Temporini ER, Kara-José N. Níveis de prevenção de problemas oftalmológicos: propostas de investigação. Arq Bras Oftalmol. 1995;58(3):189-92.

\section{Endereço para correspondência \\ José Cavalcanti Campos Júnior \\ Av. Professor Nilton Lins, $\mathbf{n}^{\circ} 500$ - Casa 27 \\ Bairro Flores \\ CEP 69058-400 - Manaus (AM), Brasil \\ E-mail: jccj@argo.com.br}

